\title{
Note on a solution to domain wall problem with the Lazarides-Shafi mechanism in axion dark matter models
}

\author{
Chandrasekhar Chatterjee, ${ }^{1,2, *}$ Tetsutaro Higaki®, ${ }^{1,3, \dagger}$ and Muneto Nitta ${ }^{1,2, \$}$ \\ ${ }^{1}$ Research and Education Center for Natural Sciences, Keio University, Yokohama 223-8521, Japan \\ ${ }^{2}$ Department of Physics, Keio University, Yokohama 223-8521, Japan \\ ${ }^{3}$ Department of Physics, Keio University, Yokohama 223-8522, Japan
}

(Received 5 April 2019; accepted 30 March 2020; published 15 April 2020)

\begin{abstract}
Axion is a promising candidate of dark matter. After the Peccei-Quinn symmetry breaking, axion strings are formed and attached by domain walls when the temperature of the universe becomes comparable to the QCD scale. Such objects can cause cosmological disasters if they are long lived. As a solution for it, the Lazarides-Shafi mechanism is often discussed through introduction of a new non-Abelian (gauge) symmetry. We study this mechanism in detail and show configuration of strings and walls. Even if Abelian axion strings with a domain wall number greater than 1 are formed in the early universe, each of them is split into multiple Alice axion strings due to a repulsive force between the Alice strings even without domain wall. When domain walls are formed as the universe cools down, a single Alice string can be attached by a single wall because a vacuum is connected by a non-Abelian rotation without changing energy. Even if an Abelian axion string attached by domain walls is created due to the Kibble Zurek mechanism at the chiral phase transition, such strings are also similarly split into multiple Alice strings attached by walls in the presence of the domain wall tension. Such walls do not form stable networks since they collapse by the tension of the walls, emitting axions.
\end{abstract}

DOI: 10.1103/PhysRevD.101.075026

\section{INTRODUCTION}

The standard model successfully explains various phenomena in experiments. However there exist several unsolved problems. One of the problems is the strong $C P$ problem. We may have a $C P$ violating term in $\mathrm{QCD}$ :

$$
\mathcal{L}=\theta \frac{g_{s}^{2}}{32 \pi^{2}} G^{a \mu \nu} \tilde{G}_{\mu \nu}^{a}
$$

where $\theta$ is a constant parameter, $g_{s}$ is the strong coupling constant, $G_{\mu \nu}^{a}$ is the gluon field strength, and $\tilde{G}_{\mu \nu}^{a}$ is its dual. Measurements of the neutron electric dipole moment give a constraint that $|\theta| \lesssim 10^{-10}$ [1], while a naive expectation is that $|\theta|=\mathcal{O}(1)$. Without any mechanisms, this would require a fine-tuning. The problem can be naturally solved if one introduces the Peccei-Quinn (PQ) mechanism with a global $U(1)$ symmetry denoted by $U(1)_{\mathrm{PQ}}$ [2-4]. After the

\footnotetext{
*chandra@phys-h.keio.ac.jp

†thigaki@rk.phys.keio.ac.jp

\#nitta@phys-h.keio.ac.jp
}

Published by the American Physical Society under the terms of the Creative Commons Attribution 4.0 International license. Further distribution of this work must maintain attribution to the author(s) and the published article's title, journal citation, and DOI. Funded by SCOAP ${ }^{3}$. spontaneous $U(1)_{\mathrm{PQ}}$ breaking, a pseudo-Nambu-Goldstone boson called the QCD axion appears. When the $U(1)_{\mathrm{PQ}}$ symmetry is explicitly broken by the QCD instanton effect via chiral anomaly between $U(1)_{\mathrm{PQ}}$ and $\mathrm{QCD}$, the axion vacuum is at a $C P$-conserving minimum of the potential and the axion solves the strong $C P$ problem. The axion decay constant $f_{a}$ is of the order of the $U(1)_{\mathrm{PQ}}$ symmetry breaking scale, and the so-called axion window is given by

$$
10^{9} \mathrm{GeV} \lesssim f_{a} \lesssim 10^{12} \mathrm{GeV}
$$

The lower bound comes from the SN 1987A neutrino burst duration [5]. The upper bound comes from the dark matter abundance by the misalignment mechanism without a tuning of the initial misalignment, in which the coherent oscillation of the axion around the vacuum accounts for the abundance [6-8]. See e.g., Refs. [9,10] for reviews.

Physics of the axion is related to the history of the universe. Below the temperature of $1 \mathrm{GeV}$, QCD instantons breaks the $U(1)_{\mathrm{PQ}}$ to $\mathbb{Z}_{N_{\mathrm{DW}}}$ symmetry, where $N_{\mathrm{DW}}$ is an integer called the domain wall number, then there appear $N_{\text {DW }}$ vacua. Along with the PQ symmetry breaking, domain walls attached to strings are formed [11-15] once one of the vacua is chosen. The cosmological scenario depends on when the breakdown of the PQ symmetry takes place. If the PQ symmetry is broken before or during 
inflation, strings and walls are inflated away because the axion field value becomes homogeneous over the scale of the Hubble horizon after inflation. They cannot affect on cosmological observations, however, there exists a stringent constraint on isocurvature perturbation produced by the axion during the inflation [16]. If the PQ symmetry is broken after inflation, such a constraint does not exist, but walls and strings may survive until late time and can affect on evolution of the universe. We will focus on the latter case in this paper.

A stability of walls attached to strings is known to depend on $N_{\text {DW }}$, which is related to a topological charge. For $N_{\mathrm{DW}}=1$, the domain wall attached to a string collapses owing to its tension, emitting axions. On top of the misalignment, axions produced by their decays accounts for a fraction of the dark matter abundance [17,18]. For $N_{\mathrm{DW}}>1$, the domain walls attached to a string constitute complex networks, which are called stringdomain wall networks. The walls in the network pull each other with their tensions and they do not shrink to a point, thus networks can be long lived. They eventually dominate the energy density of the universe beyond those of radiation and matter, and conflict with the standard cosmology. This is called a domain wall problem [14].

For a solution to the domain wall problem, several ideas have been proposed so far [19-22]. Among them, we will focus on an axion model associated with a continuous nonAbelian gauge symmetry proposed by Lazarides-Shafi [20]. ${ }^{1}$ One might think that a topologically stable domain wall is formed when $\mathbb{Z}_{N_{\mathrm{DW}}}$ symmetry is spontaneously broken by choosing the vacuum. However, when the nonAbelian symmetry is also spontaneously broken at the same time and a combination of $\mathbb{Z}_{N_{\mathrm{DW}}}$ and the broken nonAbelian rotation can make the vacuum invariant, the vacua are also continuously connected also by the non-Abelian group without changing energy. This is because the nonAbelian rotation is equivalent to a travel in the space of would-be Nambu-Goldstone (NG) modes. Then, a topological property of such a domain wall for any $N_{\mathrm{DW}}$ becomes trivial like in a case with $N_{\text {DW }}=1$. Hence the domain wall problem is solved. This is called the Lazarides-Shafi mechanism.

However, behaviors of strings and walls in the mechanism have not been discussed much in literature, while the authors of Ref. [22] discussed the decay of Abelian axion strings to Alice axion strings to solve the domain wall problem based on the mechanism. In this model, we may have $N_{\text {DW }}=2$ and a single axion string is attached by two domain walls, seemingly having a domain wall problem. The Alice string produced by the decay plays a crucial role to realize a situation similar to the $N_{\mathrm{DW}}=1 \mathrm{case}$, in which one Alice string is attached by one domain wall. Hence the

\footnotetext{
${ }^{1}$ Non-Abelian global symmetries are also viable for solving the domain wall problem [23].
}

network is unstable in the presence of the domain wall tension. (See Refs. [24,25] for Abelian string and also Refs. [26,27] for reviews of cosmic strings.)

Alice strings have a peculiar property that when the electric charge of a charged particle encircles around an Alice string, it changes its sign [28,29]. Other peculiar properties, such as a topological obstruction, a nonlocal charge called the Chesire charge, and non-Abelian statistics, have been studied in the literature [30-37]. While a typical Alice string is present in an $S O(3)$ gauge theory with scalar fields in the fiveplet representation (a traceless symmetric tensor), recently it has been found that a $U(1) \times$ $S O(3)$ gauge theory with complex triplet scalar fields also admits an Alice string, which is a Bogomol'nyi-PrasadSommerfield state $[38,39]$ and is stable, thereby possible to be embedded into supersymmetric theories [40,41]. A global analog was known in the context of Bose-Einstein condensates in condensed matter physics [42-45]. The Alice axion string proposed in Ref. [22] is the case that only the $U(1)$ part is global identified with an axion, while the $S U(2)$ part is a gauge symmetry.

In this paper, we show why the domain wall problem is solved physically in more detail, focusing on dynamics of domain walls and two types of axion strings. It is found that even if Abelian axion strings are formed in the early universe, the string decays into multiple Alice axion strings owing to a repulsive force between the Alice strings. When domain walls are formed at the chiral phase transition as the universe cools down, a single Alice axion string is attached by a single wall because a vacuum is connected by a nonAbelian rotation without changing energy. Such walls do not form stable networks since they collapse owing to the tension of the walls, emitting axions similarly to the $N_{\text {DW }}=1$ case. Also, at the chiral phase transition, two types of domain walls may be created by the Kibble-Zurek mechanism [46,47], and can be glued along an Abelian axion string. The Abelian axion string is pulled by these domain walls and is split into multiple Alice axion strings, each of which is attached by one domain wall. In either of these cases, the domain wall problem can be solved.

The rest of this paper is organized as follows. In Sec. II, we briefly review the QCD axion and domain wall problem. In Sec. III, we introduce an axion model with heavy quarks and a new gauge symmetry for solving the domain wall problem. In Sec. IV, properties of strings and domain walls are studied. In Sec. V, we study domain walls attached to strings. Section VI is devoted to discussion and conclusions.

\section{REVIEW OF THE QCD AXION AND DOMAIN WALL PROBLEM}

In this section, we review the QCD axion based on the Kim-Shifman-Vainstein-Zakharov (KSVZ) model $[48,49]$ and domain wall problem for simplicity. (The Dine-Fischler-Srednicki-Zhitnitsky model $[50,51]$ can be 
also discussed in a similar way.) Let us consider a coupling

$$
\mathcal{L}=y \bar{Q}^{i} \Phi Q^{i}+\text { H.c. } \quad\left(i=1,2, \ldots, N_{\mathrm{DW}}\right) .
$$

Here, $(\bar{Q}, Q)$ are $N_{\text {Dw }}$ pairs of extra heavy quarks in the $S U(3)_{c}$ gauge symmetry, and $\Phi$ is a complex scalar singlet under the Standard Model gauge symmetry. This coupling is invariant under the global $U(1)_{\mathrm{PQ}}$ symmetry:

$$
\bar{Q} \rightarrow e^{-i \theta} \bar{Q}, \quad Q \rightarrow Q, \quad \Phi \rightarrow e^{i \theta} \Phi .
$$

Here $\theta$ is a transformation parameter. Suppose that after inflation $\Phi$ develops vacuum expectation value (VEV) $\eta$ and the $U(1)_{\mathrm{PQ}}$ is spontaneously broken down. Thus the scalar field is parametrized as

$$
\Phi=\sqrt{2}\left(\eta+\frac{\sigma}{2}\right) \exp \left(i \frac{a}{2 \eta}\right)
$$

Here $\sigma$ is supposed to be stabilized and we neglect it throughout this paper, $a$ is the QCD axion. Note that a $U(1)_{\mathrm{PQ}}$ rotation of $a \rightarrow a+2 \pi \cdot(2 \eta)$ is a symmetry. All pairs of extra quarks obtain heavy mass $\sqrt{2} y \eta \times e^{i \frac{a}{2 \eta}}$. After integrating out extra quarks with the rotation of $\bar{Q} Q \rightarrow$ $e^{-i \frac{a}{2 \eta}} \bar{Q} Q$, we obtain

$$
\mathcal{L}=\frac{a}{f_{a}} \frac{g_{s}^{2}}{32 \pi^{2}} G^{a \mu \nu} \tilde{G}_{\mu \nu}^{a}, \quad \text { where } f_{a} \equiv \frac{2 \eta}{N_{\mathrm{DW}}}
$$

via the chiral anomaly between $U(1)_{\mathrm{PQ}}$ and $S U(3)_{c}$. Thus $\langle a\rangle=0$ means the $C P$-conserving vacuum. After the chiral symmetry breaking in QCD, gluons and light quarks are integrated out, and the axion potential can be written as

$$
V(a) \simeq m_{\pi}^{2} f_{\pi}^{2}\left(1-\cos \left(\frac{a}{f_{a}}\right)\right) \sim \cos \left(N_{\mathrm{DW}} \frac{a}{2 \eta}\right) .
$$

Here, $m_{\pi}$ is the pion mass and $f_{\pi}$ is the pion decay constant. As desired, $\langle a\rangle=0$ is obtained in the vacuum and the strong $C P$ problem is then solved. The axion mass is given by

$$
m_{a} \simeq \frac{m_{\pi} f_{\pi}}{f_{a}} \simeq 6 \times 10^{-4} \mathrm{eV}\left(\frac{10^{10} \mathrm{GeV}}{f_{a}}\right) .
$$

Note that $a \rightarrow a+2 \pi f_{a}$ is the $\mathbb{Z}_{N_{\mathrm{DW}}}$ symmetry against the above potential, in addition to the original larger symmetry of $a \rightarrow a+2 \pi N_{\mathrm{DW}} f_{a}$. Thus, there exist $N_{\mathrm{DW}}$ vacua. Once one of the vacua is chosen, $\mathbb{Z}_{N_{\mathrm{DW}}}$ symmetry is spontaneously broken and topologically stable domain walls (attached to Abelian strings) appear between vacua for $N_{\text {DW }}>1$. When one classically travels from a vacuum to the next one in the axion field space, it is necessary to climb the potential energy. The walls pull each other with their tensions and they do not shrink to a point, thus can be long lived. The presence of stable domain walls conflict with the standard cosmology, because they eventually dominate the energy density of the universe beyond those of radiation and matter. It is verified in simulations that domain walls survive until late time for $N_{\mathrm{DW}}>1$, while they decay for $N_{\text {DW }}=1$ or in the presence of bias potential for $N_{\text {DW }}>1$ $[17,18]$. For $N_{\mathrm{DW}}=1$, the axion dark matter abundance produced by decays of walls and strings is estimated as

$$
\Omega_{a} h^{2} \sim 10^{-2}\left(\frac{f_{a}}{10^{10} \mathrm{GeV}}\right)^{1.19} .
$$

\section{THE MODEL WITH A NON-ABELIAN GAUGE SYMMETRY}

Following Ref. [22], we explain the model to implement the Lazarides-Shafi mechanism in the KSVZ case. We shall start with the hidden $S U(2)_{H}$ gauge theory on top of the global $U(1)_{\mathrm{PQ}}$ symmetry. The Lagrangian is given as

$\mathcal{L}=-\frac{1}{2} \operatorname{Tr} F_{\mu \nu} F^{\mu \nu}+\operatorname{Tr}\left|D_{\mu} \Phi\right|^{2}-V(\Phi)+(y \bar{Q} \Phi Q+$ H.c. $)$,

where $F_{\mu \nu}$ is the $S U(2)_{H}$ gauge field strength, $\Phi$ is a complex adjoint scalar field and $(\bar{Q}, Q)$ are extra quarks charged also under both $S U(2)_{H}$ and $U(1)_{\mathrm{PQ}}{ }^{2}$ Their charge assignment against $\left(S U(3)_{c}, S U(2)_{H}, U(1)_{\mathrm{PQ}}\right)$ is as follows: $\Phi:(1,3,1), \bar{Q}:(\overline{3}, \overline{2},-1)$ and $Q:(3,2,0) . S U(2)_{H}$ adjoint fields can be expanded with the $S U(2)$ generators $\tau^{a}=\frac{1}{2} \sigma^{a}$ as $\Phi=\phi^{a} \tau^{a}$ and $A_{\mu}=A_{\mu}^{a} \tau^{a}$, and the covariant derivative is defined as $D_{\mu} \Phi=\partial_{\mu} \Phi-i g\left[A_{\mu}, \Phi\right]$. Here $g$ is the $S U(2)_{H}$ gauge coupling. The potential for $\Phi$ is given as

$$
V(\Phi)=\frac{\lambda_{1}}{2}\left(\operatorname{Tr}\left(\Phi^{\dagger} \Phi\right)-2 \eta^{2}\right)^{2}+\frac{\lambda_{2}}{2} \operatorname{Tr}\left(\left[\Phi, \Phi^{\dagger}\right]^{2}\right) .
$$

This is a usual potential for complex adjoint scalar field that breaks $U(1)_{\mathrm{PQ}} \times S U(2)_{H}$ spontaneously. Later we will consider an explicit violation term for $U(1)_{\mathrm{PQ}}$, which is relevant to axion mass and domain wall construction. The ground state is given by the solution of the equations

$$
\operatorname{Tr} \Phi^{\dagger} \Phi=2 \eta^{2}, \quad\left[\Phi, \Phi^{\dagger}\right]=0 .
$$

The vacuum solution we may generally choose as

$$
\langle\Phi\rangle=2 \eta \tau^{1}=\eta \sigma^{1}
$$

\footnotetext{
${ }^{2}$ To avoid overproduction of massive particles at a high temperature, the $S U(2)_{H}$ doublet scalar field with $\mathrm{TeV}$ mass is introduced. As a consequence, extra quarks need to be charged under $U(1)_{Y}$. Further, there may exist observational signals, but we will focus only on configurations of strings and walls.
} 
The VEV breaks $U(1)_{\mathrm{PQ}}$ and $S U(2)_{H}$, then there exists the unbroken $U(1)_{H}$ gauge symmetry with a generator of $\tau^{1}$. This vacuum is invariant under $O(2)$ rather than $S O(2)$. The elements are given as

$$
H=\left\{\left(1, e^{i \alpha \tau^{1}}\right),\left(-1, i\left(c_{1} \sigma^{2}+c_{2} \sigma^{3}\right) e^{i \alpha \tau^{1}}\right)\right\} .
$$

Here $\alpha, c_{1,2}$ are parameters and the condition of $c_{1}^{2}+c_{2}^{2}=$ 1 is satisfied. The first entry of each element of $H$ is $U(1)_{\mathrm{PQ}}$ and the second entry is the element of $U(1) \subset S U(2)_{H}$ group, which can act as adjoint representation on $\langle\Phi\rangle$. The first element is the usual element of $U(1)_{H} \subset O(2)$ connected to identity element $(1,1)$. However, the second element is a nontrivial $\mathbb{Z}_{2}$. Note that $e^{i \pi Q_{\mathrm{PQ}}}=-1$ for $Q_{\mathrm{PQ}}=1$, where $Q_{\mathrm{PQ}}$ is the PQ charge of the $\Phi$, and $e^{i \pi \tau^{a}}=$ $i \sigma^{a}$ for $a=2,3$ : The first entry is $\pi$ rotation of $U(1)_{\mathrm{PQ}}$ and the part of second entry $i\left(c_{1} \sigma^{2}+c_{2} \sigma^{3}\right)$ gives $\pi$ rotation of broken $S U(2)_{H}$ around an axis orthogonal to $\tau^{1}$. So the second element of $H$ is referred to as the disconnected elements of $O(2)$.

More specifically we may describe the symmetry breaking procedure as

$$
\begin{aligned}
G & =U(1)_{\mathrm{PQ}} \times \frac{S U(2)_{H}}{\mathbb{Z}_{2}} \simeq U(1)_{\mathrm{PQ}} \times S O(3)_{H} \\
\longrightarrow H & =\mathbb{Z}_{2} \ltimes U(1)_{H} \simeq O(2),
\end{aligned}
$$

where $\ltimes$ denotes the semidirect product because the $\mathbb{Z}_{2}$ caused by $\pi$ rotation in the above discussion does not commute with the unbroken $U(1)_{H}$ generated by $\tau^{1}$. The vacuum manifold ${ }^{3}$ is found to be

$$
\frac{G}{H}=\frac{U(1)_{\mathrm{PQ}} \times S O(3)}{O(2)}=\frac{S^{1} \times S^{2}}{\mathbb{Z}_{2}} .
$$

The fundamental group is $\pi_{1}(G / H)=\mathbb{Z}$, and this shows the existence of strings. It is noted that $\Phi$ has 6 degrees of freedom. In this vacuum, two of three NG modes associated with the broken $S U(2)_{H}$ are eaten by the gauge fields $A^{2}$ and $A^{3}$, which get massive. The rest (pseudo)NG mode relevant to $U(1)_{\mathrm{PQ}}$ is the $\mathrm{QCD}$ axion denoted by $a$. The remaining three modes of $\Phi$ also become massive in the vacuum [22].

By integrating out extra heavy quarks with a mass matrix of

$$
\Phi=2 \eta e^{i a / 2 \eta} \tau^{1}
$$

we have

\footnotetext{
${ }^{3} \mathbb{Z}_{2}$ in the original $S U(2)_{H}$ is the center acting on $\Phi$ trivially. Even though there exist doublets, the vacuum manifold does not change unless they develop VEVs.
}

$\mathcal{L}=N_{\mathrm{DW}} \frac{a}{2 \eta} \frac{g_{s}^{2}}{32 \pi^{2}} G^{a \mu \nu} \tilde{G}_{\mu \nu}^{a}+N_{\mathrm{DW}}^{\prime} \frac{a}{2 \eta} \frac{g^{2}}{32 \pi^{2}} F^{\prime \mu \nu} \tilde{F}_{\mu \nu}^{\prime}$.

Here, $N_{\mathrm{DW}}=2$ and $N_{\mathrm{DW}}^{\prime}=3$, and $F^{\prime \mu \nu}$ is the unbroken $U(1)_{H}$ gauge field strength. After the chiral symmetry breaking in QCD, the axion potential can be written as

$$
\begin{aligned}
V(a) & \simeq m_{\pi}^{2} f_{\pi}^{2}\left(1-\cos \left(N_{\mathrm{DW}} \frac{a}{2 \eta}\right)\right) \\
& \equiv m_{a}^{2} f_{a}^{2}\left(1-\cos \left(\frac{a}{f_{a}}\right)\right) .
\end{aligned}
$$

Here, $m_{a} \simeq m_{\pi} f_{\pi} / f_{a}$ and $f_{a}=\frac{2 \eta}{N_{\mathrm{DW}}}$. To study domain wall later, we parametrize this potential with $\Phi$ as

$$
V_{\mathrm{DW}}(\Phi)=\mu\left(\operatorname{Det} \Phi+\operatorname{Det} \Phi^{\dagger}\right)+\text { const. }
$$

Here $\mu$ is assumed to be of $\mathcal{O}\left(m_{a}^{2} / N_{\text {DW }}^{2}\right)$ constant. ${ }^{4}$ A constant term is added to make the vacuum energy positive definite. This form is motivated by the fact that axion and walls do not appear for $\Phi=0$. (See also [52].) $V_{\mathrm{DW}}(\Phi)$ will be added to $V(\Phi)$ of Eq. (11) in the following sections about domain walls. Since $N_{\mathrm{DW}}=2$ vacua are connected by $\mathbb{Z}_{2}$ embedded both in the $U(1)_{\mathrm{PQ}}$ and the broken $S U(2)_{H}$, which acts as $\langle\Phi\rangle \rightarrow-\langle\Phi\rangle$, the domain wall problem is solved as seen later. For a general $N_{\mathrm{DW}}$, a model with an $S U\left(N_{\mathrm{DW}}\right)_{H}$ (gauge) symmetry is viable to implement the Lazarides-Shafi mechanism, because $\mathbb{Z}_{N_{\mathrm{DW}}}$ of the center in $S U\left(N_{\mathrm{DW}}\right)_{H}$ connects $N_{\mathrm{DW}}$ vacua.

Now, we have also monopole since $\pi_{2}(G / H)=\mathbb{Z}$. It can be also a candidate of dark matter with mass of $10^{10} \mathrm{GeV}$ which is supposed to be created by the first order phase transition of the $S U(2)_{H}$ at a high temperature around $3 \times 10^{9} \mathrm{GeV}$ [22]. The monopole can become a dyon with an electric $U(1)_{H}$ charge via the Witten effect [53] when the axion cannot cancel the $C P$ phase in the hidden sector $\theta$ term. Then the monopole may have minielectric charge via kinetic mixing between the photon and hidden photon in the $U(1)_{H}$. We assume that the minicharge is sufficiently small to evade experimental bounds $[54,55]$. The monopole will not give further effects to the solution to the domain wall problem in the KSVZ case. For $N_{\mathrm{DW}}>2$ with $S U(2)_{H}$, however, the Witten effect to the axion mass in the early universe [56] can give a significant effect to solve the domain wall problem in the presence of monopoles $[21,22] .^{5}$

\footnotetext{
${ }^{4} \mu$ actually depends on temperature of the universe via QCD instanton. We will focus on a period during when domain walls are formed below a temperature lower than $1 \mathrm{GeV}$ around which the axion mass is close to that at zero temperature.

${ }^{5}$ The monopole can also play an important role in suppressing isocurvature perturbation of the axion generated during inflation $[21,57,58]$, if the PQ breaking occurs before/during the inflation.
} 


\section{CONSTRUCTION OF STRINGS}

Since the fundamental group of the vacuum manifold is an integer so we may expect topological string solutions. In this section we discuss two kinds of string solutions. One is an Abelian axion string originating from $U(1)_{\mathrm{PQ}}$ symmetry breaking and this kind of strings can be thought of as usual global Abelian strings. Another is nontrivial Alice axion strings which contain non-Abelian magnetic flux. This string can be classified by the elements of the unbroken group $H=O(2)$. In the following, we discuss the splitting of one Abelian axion string into two Alice axion strings. We show full numerical results of the splitting and will set $V_{\text {DW }}(\Phi)=0$ in this section.

\section{A. Abelian axion strings}

Here we would like to discuss the fully Abelian axion string which results from the $U(1)_{\mathrm{PQ}}$ breaking. The axisymmetric ansatz for this string is given by

$$
\Phi=2 \eta f(r) e^{i m \theta} \tau^{1}, \quad A_{\mu}=0,
$$

where $m$ is winding number, $r$ and $\theta$ are radial coordinate and azimuthal angle respectively. The boundary condition is given by $f(0)=0, f(\rho)=1$, where $\rho$ is the system size in the radial direction. The profile function $f(r)$ can be calculated from the axisymmetric equations of motion with this boundary condition. After inserting the above ansatz for $m=1$, we easily find the static Hamiltonian for $\Phi$ with Eq. (10). The two-dimensional integral of the Hamiltonian density is given by

$\mathcal{H}^{(2)}=\int d^{2} x 2 \eta^{2}\left\{f^{\prime}(r)^{2}+\left(\frac{f(r)}{r}\right)^{2}+\lambda_{1} \eta^{2}\left(f(r)^{2}-1\right)^{2}\right\}$.

Here prime denotes the derivative with respect to $r$. Note that because the above Hamiltonian is written in a static case we have $\mathcal{H}^{(2)}=-L$, where $L=\int d^{2} x \mathcal{L}$ is the twodimensional integral of the Lagrangian Eq. (10) with the ansatz. With the radial coordinate, the one-dimensional Euler-Lagrange equation of the profile function reads

$$
-\frac{1}{r} \frac{d}{d r} r \frac{d f(r)}{d r}+\frac{1}{r^{2}} f(r)+2 \lambda_{1} \eta^{2}\left(f(r)^{2}-1\right) f(r)=0 .
$$

The numerical result is shown in Fig. 1, while the analytical solution of the above equation is not known. The tension (energy per unit length) of these strings is found from the above $\mathcal{H}^{(2)}$. The approximate value of the tension far from the string core is easy to compute if we insert the ansatz at a large distance into the Hamiltonian:

$$
T_{\mathrm{PQ}}=\mathcal{H}^{(2)} \simeq \int d^{2} x \operatorname{Tr}\left|\partial_{i} \Phi\right|^{2} \sim 2 \pi \times 2 \eta^{2} \times m^{2} \log \rho .
$$

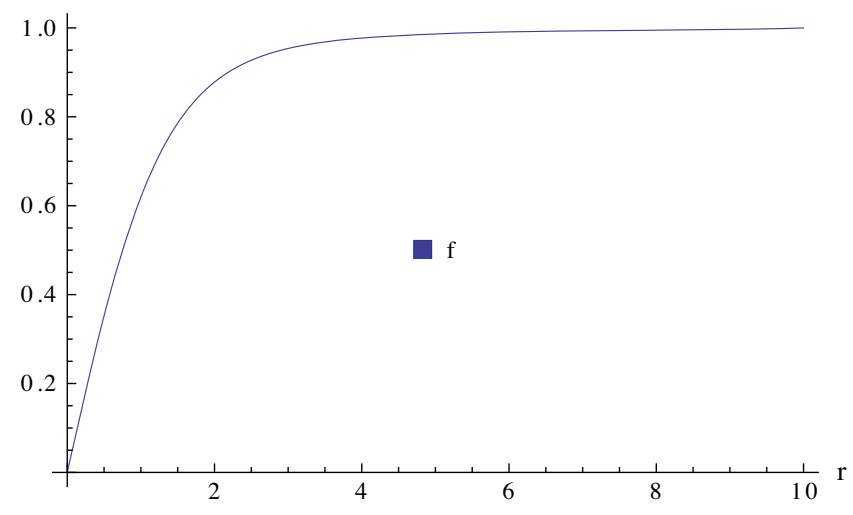

FIG. 1. Radial dependence of the profile function of $f(r)$ for $m=1, \eta=2$ and $\lambda_{1}=0.2$.

We may notice that energy is logarithmically divergent and energy depends on the square of the winding number $m^{2}$. The energy stored inside of the core is estimated as of order $\eta^{2}$, to which the scalar potential contributes, and can be neglected at a large distance. It is always energetically favorable to decay the higher winding number string into lower winding strings. Since Alice axion strings have $m=1 / 2$ as seen later, so an Abelian axion string with $m=1$ decays into two Alice axion strings.

\section{B. Alice axion strings}

The Alice axion string is a kind of topological string which changes the sign of electric charge of a probe particle with an original gauge symmetry after one encirclement around the string. In our case, the generator of the unbroken $U(1)_{H}$ changes its sign with the $S U(2)_{H}$ after one rotation. This is because a particle charged under the $U(1)_{H}$ is affected by the broken $S U(2)_{H}$ flux inside the Alice string. To understand this better, let us first consider the field value rotating around an Alice axion string, which depends on the azimuthal angle at a large distance $\rho$,

$$
\begin{aligned}
\Phi(\rho, \theta) & \sim \eta e^{i \frac{\theta}{2}}\left(\begin{array}{cc}
0 & e^{i \frac{\theta}{2}} \\
e^{-i \frac{\theta}{2}} & 0
\end{array}\right) \equiv e^{i \frac{i}{2}} \Omega(\theta) \Phi(\rho, 0) \Omega^{-1}(\theta), \\
A_{i} & \sim-\frac{1}{2 g} \frac{\epsilon_{i j} x^{j}}{\rho^{2}} \tau^{3}(i, j=1,2), \quad A_{0}=A_{3}=0 .
\end{aligned}
$$

Here, $x^{2}+y^{2}=r^{2}, \Phi(\rho, 0)=2 \eta \tau^{1}=\langle\Phi\rangle$ and the holonomy $\Omega(\theta)$ rotating the $\Phi(\rho, 0)$ by $\theta$ can be defined by the broken $S U(2)_{H}$ gauge field:

$$
\Omega(\theta)=P e^{i g \int_{0}^{\theta} \mathbf{A} \cdot \mathbf{d} \mathbf{l}}=e^{i \frac{\theta}{2} \tau^{3}} \in S U(2)_{H},
$$

where we used $\partial_{i} \theta=-\epsilon_{i j} x^{j} / r^{2}$. It is easy to compute the (non-Abelian) flux in the broken $S U(2)_{H}$ trapped inside the string: 


$$
\text { Flux }=\oint \mathbf{A} \cdot \mathbf{d} \mathbf{l}=\frac{\pi}{g} \tau^{3}
$$

This $S U(2)$ holonomy can also be $\Omega(\theta)=e^{i \frac{\theta}{2} \tau^{2}}$ or more generally $\Omega(\theta)=e^{i \frac{i}{2} \hat{n}} \quad$ with $\quad \hat{n}=\sin \alpha \tau^{2}+\cos \alpha \tau^{3}=$ $e^{i \alpha \tau^{1}} \tau^{3} e^{-i \alpha \tau^{1}}$. Correspondingly, the flux is along $\hat{n}$. The presence of $\alpha$ can be understood as follows. The unbroken symmetry $U(1)_{H}$ of the vacuum is generated by $\tau_{1}$. However, this $U(1)_{H}$ acts on the Alice string solution with a parameter $\alpha$. Namely, the Alice string configuration spontaneously breaks $U(1)_{H}$ symmetry of the vacuum, implying the appearance of a Nambu-Goldstone mode in the vicinity of the Alice string. Therefore, $\alpha$ parametrizes a continuous family of the Alice string solution of the same energy, and is a $U(1)$ modulus of the Alice string [41].

While $\langle\Phi\rangle$ in Eq. (13) is invariant under the $U(1)_{H}$ with $\tau^{1}, \Phi(\rho, \theta)$ in Eq. (25) is not invariant under such unbroken elements in Eq. (14) because the unbroken transformation for $\Phi(\rho, \theta)$ becomes angle dependent. The $U(1)_{H}$ generator must be changed by the holonomies with the gauge field when it goes around the Alice string as

$$
Q_{\theta}=\Omega(\theta) Q_{0} \Omega(\theta)^{-1}
$$

Here $Q_{0} \propto \tau^{1}$, thus transformations with $Q_{\theta}$ make $\Phi(\rho, \theta)$ invariant as $e^{i \beta Q_{\theta}} \Phi(\rho, \theta) e^{-i \beta Q_{\theta}}=\Phi(\rho, \theta)$, where $\beta$ is a transformation parameter. After encircling a full loop around the Alice string, we find that $\Omega(2 \pi)=e^{i \pi \tau^{3}} \in$ $\mathbb{Z}_{2} \subset H$, hence the unbroken generator becomes

$$
Q_{2 \pi}=-Q_{0}
$$

This is nothing but the most characteristic property of the Alice string; the charge of a charged particle flips its sign when it encircles around an Alice string.

To find a solution of the Alice axion string, we shall consider an axisymmetric ansatz as

$$
\begin{aligned}
\Phi(r, \theta) & =\eta\left(\begin{array}{cc}
0 & f_{1}(r) e^{i \theta} \\
f_{2}(r) & 0
\end{array}\right), \\
A_{i} & =-\frac{1}{2 g} \frac{\epsilon_{i j} x^{j}}{r^{2}} a(r) \tau^{3}(i, j=1,2), \\
A_{0} & =A_{3}=0,
\end{aligned}
$$

where $f_{1,2}(r)$ and $a(r)$ are profile functions of the scalar fields and gauge field with the boundary condition that $f_{1}(0)=f_{2}(0)=0, f_{1}(\rho)=f_{2}(\rho)=1, a(0)=0, a(\rho)=1$. After inserting the above ansatz, two-dimensional integration of the static Hamiltonian density can be expressed as ${ }^{6}$

$$
\begin{aligned}
\mathcal{H}^{(2)} & =\int d^{2} x\left[\frac{1}{2} \operatorname{Tr} F_{i j}^{2}++\operatorname{Tr}\left|D_{i} \Phi\right|^{2}+V(\Phi)\right] \\
& =2 \pi \int r d r\left[\eta^{2}\left\{f_{1}^{\prime 2}+f_{2}^{\prime 2}+\frac{f_{1}^{2}}{r^{2}}\left(1-\frac{a}{2}\right)^{2}+\frac{f_{2}^{2}}{r^{2}} \frac{a^{2}}{4}+\frac{\eta^{2} \lambda_{1}}{2}\left(f_{1}^{2}+f_{2}^{2}-2\right)^{2}+\eta^{2} \lambda_{2}\left(f_{1}^{2}-f_{2}^{2}\right)^{2}\right\}+\frac{1}{8 g^{2}} \frac{a^{\prime 2}}{r^{2}}\right] .
\end{aligned}
$$

As in the Abelian string case, the equations of motion of the profile functions read

$$
\begin{aligned}
- & \frac{1}{r} \frac{d}{d r}\left(r \frac{d}{d r} f_{1}(r)\right)+\frac{1}{r^{2}}\left(1-\frac{a}{2}\right)^{2} f_{1}(r)+\lambda_{1} \eta^{2}\left(f_{1}(r)^{2}+f_{2}(r)^{2}-2\right) f_{1}(r)+2 \eta^{2} \lambda_{2}\left(f_{1}^{2}-f_{2}^{2}\right) f_{1}(r)=0 \\
& -\frac{1}{r} \frac{d}{d r}\left(r \frac{d}{d r} f_{2}(r)\right)+\frac{1}{r^{2}} \frac{a^{2}}{4} f_{2}(r)+\lambda_{1} \eta^{2}\left(f_{1}(r)^{2}+f_{2}(r)^{2}-2\right) f_{2}(r)-2 \eta^{2} \lambda_{2}\left(f_{1}^{2}-f_{2}^{2}\right) f_{2}(r)=0 \\
& -r \frac{d}{d r}\left(\frac{1}{r} \frac{d}{d r} a(r)\right)+4 g^{2} \eta^{2} f_{1}(r)^{2}\left(1-\frac{a}{2}\right)+2 g^{2} \eta^{2} f_{2}(r)^{2} a(r)=0
\end{aligned}
$$

The profile functions are solved numerically for several values of $\lambda_{1}$ and $\lambda_{2}$, and plotted in Fig. 2. It is noted that in the equation of motion for $f_{2}$ the potential contribution can vanish if $\lambda_{1}=2 \lambda_{2}$ is taken.

\footnotetext{
${ }^{6}$ Although in the Hamiltonian there is no $1 / g^{2}$ term in front of the gauge kinetic term, ansatz of the gauge field includes $1 / \mathrm{g}$. After all, we have the gauge coupling dependence only on the gauge kinetic term.
}

The tension far from the string core can be approximately computed with the ansatz at a large distance $\rho$ in Eq. (25):

$$
T_{\text {Alice }}=\mathcal{H}^{(2)} \simeq \int d^{2} x \operatorname{Tr}\left|\mathcal{D}_{i} \Phi\right|^{2} \simeq \frac{\pi}{2} \times 2 \eta^{2} \log \rho
$$

It should be noted that the energy of the Alice axion string is also logarithmically divergent and the same as that of the 


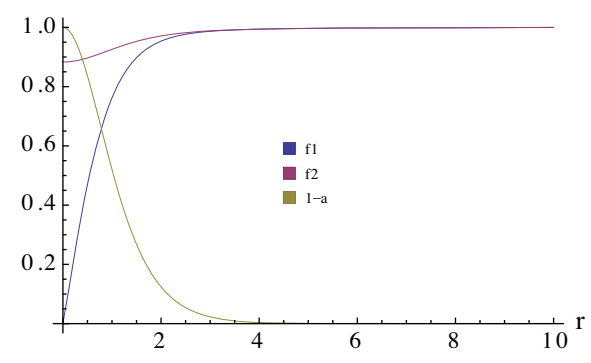

(a)

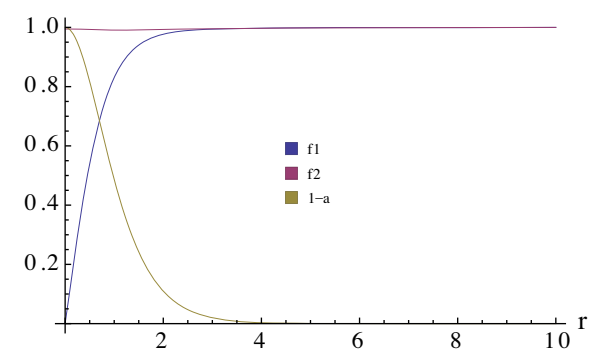

(b)

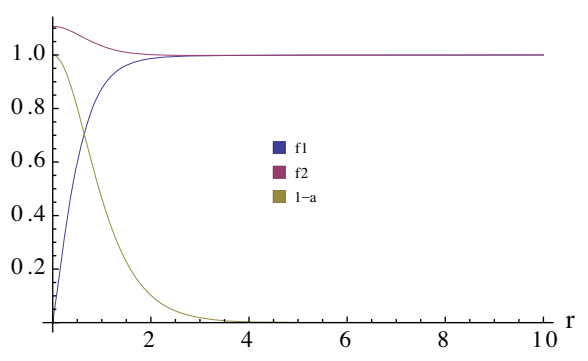

(c)

FIG. 2. Radial dependence of the profile functions of $f_{1}(r), f_{2}(r)$ and $a(r)$ for $g=0.5$ and $\eta=2$. The remaining parameters are chosen as: (a) $\lambda_{1}=0.2$ and $\lambda_{2}=0.2\left(\frac{\lambda_{1}}{\lambda_{2}}=1<2\right)$, (b) $\lambda_{1}=0.4$ and $\lambda_{2}=0.2\left(\frac{\lambda_{1}}{\lambda_{2}}=2\right)$, and (c) $\lambda_{1}=0.6$ and $\lambda_{2}=0.2\left(\frac{\lambda_{1}}{\lambda_{2}}=3>2\right.$. This is used also in Sec. V.

Abelian axion string with $m=1 / 2$ in Eq. (24). Here there would be a contribution from the $S U(2)$ magnetic field $\frac{1}{2} \operatorname{Tr} F_{i j}^{2}$, however that we have neglected due to the logarithmic divergence of the leading term and the energy stored inside the core of the Alice string is estimated as of order $\eta^{2}$, to which fluxes and the scalar potential contribute. As mentioned in the previous subsection, an Abelian axion string (with $m=1$ ) can decay into two Alice axion strings (with $m=1 / 2$ ), since the energy of the Abelian strings gets lower by the decay: $1^{2}>(1 / 2)^{2}+(1 / 2)^{2}=1 / 2$ in the $m=1$ string tension unit, whereas the winding number is conserved. The remaining $1 / 2$ energy is thought to be converted to axions at the decay, and they will contribute to a fraction of the final abundance of the axion.

\section{The decay of an Abelian axion string to two Alice axion strings}

In this subsection, we try to understand the decay of the Abelian axion string to the Alice axion strings. As seen above, the tension for the Abelian string is different from the Alice strings by a factor 4 . So the Abelian string (with $m=1$ ) is always energetically favorable to decay into two Alice strings (with $m=1 / 2$ ) with the conserved winding number. These two Alice axion strings produced by the decay must have opposite flux direction, since the parent Abelian axion string contains no flux and a field configuration at a large distance does not change through the decay. The configuration of $\Phi$ at an angle $\theta$ and a large distance $\rho$, which is far from the string core, can be in general written by

$$
\begin{aligned}
\Phi(\rho, \theta) & =h_{0} h\langle\Phi\rangle h^{\dagger}, \\
\Omega & =\left\{\left(h_{0}, h\right) \mid h_{0} \in U(1)_{\mathrm{PQ}},\right. \\
h & \left.=P e^{i g \int_{0}^{\theta} \mathbf{A} \cdot \mathbf{d l}} \in S U(2)_{H}\right\}, \\
\Phi(\rho, 2 \pi) & =\Phi(\rho, 0)=\langle\Phi\rangle=2 \eta \tau^{1},
\end{aligned}
$$

where $\Omega=\Omega(\theta)$ is a path dependent rotation matrix with two entries around the strings in the axisymmetric case. For an Abelian string, the gauge field is vanishing, whereas for an Alice string with the positive flux the gauge field is given by the ansatz in the previous subsection. For a full loop we have $\Omega(2 \pi)=\left(h_{0}(2 \pi), h(2 \pi)\right) \in H$ of $O(2)$, where $h(2 \pi)=P e^{i g \oint \mathbf{A} \cdot \mathbf{d l}}$. For a rotation around the Abelian axion strings by $\theta, \Omega^{0}(\theta)=\left(e^{i \theta}, 1\right)$ and $\Omega^{0}(2 \pi)=$ $(1,1) \in H$, where zero denotes for one rotation around the Abelian axion string. For a rotation by $\theta$ around a single axisymmetric Alice axion string with a positive flux, $\Omega^{+}(\theta)=\left(e^{i \frac{\theta}{2}}, e^{i \frac{\theta}{2} \tau^{3}}\right)$ and $\Omega^{+}(2 \pi)=\left\{-1, i \sigma^{3}\right\} \in H$. This is similar to $\Omega^{-}$for the Alice string with a negative flux: $\Omega^{-}(\theta)=\left(e^{i \frac{\theta}{2}}, e^{-i \frac{\theta}{2} \tau^{3}}\right)$ and $\Omega^{-}(2 \pi)=\left\{-1,-i \sigma^{3}\right\} \in H$.

Now let us understand what happens at the decay of the Abelian axion string to two Alice axion strings. Just at the time when a single Abelian axion string is split into two Alice strings, the boundary condition remains unchanged because it is understood with $\Omega$. To show it, we draw a loop $C$ around the Abelian axion string before splitting. In this case, the $\Omega$ is given by $\Omega^{0}(C)=(1,1)$. Just after the splitting, we divide the loop in two parts as $C=C_{1}+C_{2}$ and we close the loops by connecting the points $a$ and $b$ by the path $R$ as shown in Fig. 3. In this case we have

$$
\begin{aligned}
\Omega^{-}\left(C_{1}+R\right) & =\left(h_{0}\left(C_{1}+R\right)=e^{i \pi},\right. \\
h\left(C_{1}+R\right) & \left.=P e^{i g \oint_{C_{1}+R} \mathbf{A} \cdot \mathbf{d l}}\right) \\
\Omega^{+}\left(C_{2}-R\right) & =\left(h_{0}\left(C_{2}-R\right)=e^{i \pi},\right. \\
h\left(C_{2}-R\right) & \left.=P e^{i g \oint_{C_{2}-R} \mathbf{A} \cdot \mathbf{d l}}\right) .
\end{aligned}
$$

So we find

$$
\Omega^{0}(C)=\Omega^{+}\left(C_{2}-R\right) \Omega^{-}\left(C_{1}+R\right)=\left(1, P e^{i g \oint_{C} \mathbf{A} \cdot \mathbf{d l}}=1\right) .
$$

This shows that field configurations at a large distance do not change, while the splitting takes place. 

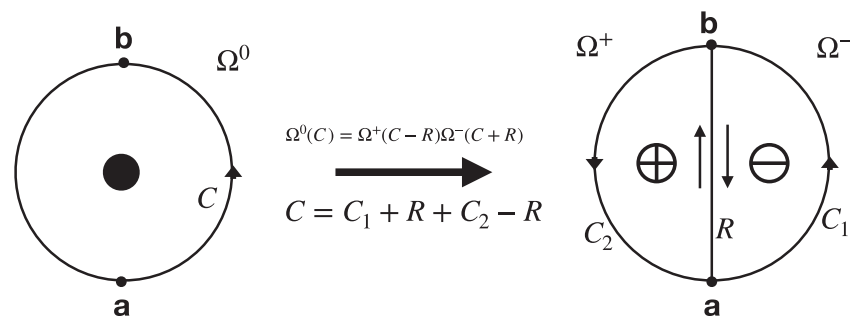

FIG. 3. Loops around the strings. Left: A loop $C$ around the Abelian axion string. Right: A loop $C$ is decomposed to $C_{1}+R$ and $C_{2}-R$, where the former is a loop around the Alice axion string with the negative flux and the latter is a loop around the Alice string with the positive flux.

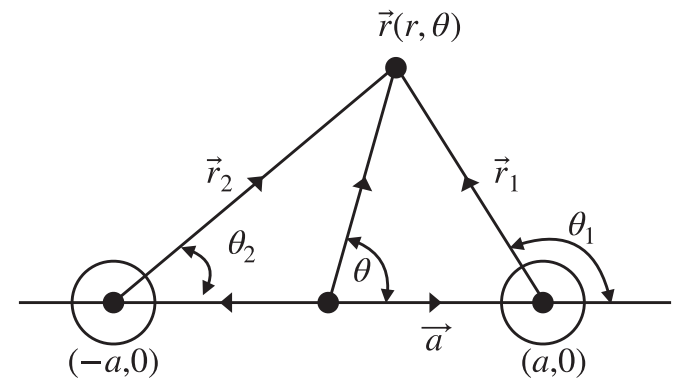

FIG. 4. A configuration of two Alice axion strings. One Alice string with the negative flux is at $(a, 0)$ in the $x-y$ plane, whereas another with the positive flux is at $(-a, 0) .\left|\vec{r}_{1,2}\right|$ are the distance vectors from the two Alice strings from the point $\vec{r}=(r, \theta)$, and $\theta_{1,2}$ are the angles around them.

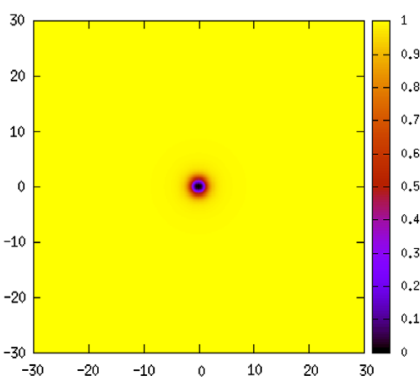

(a)

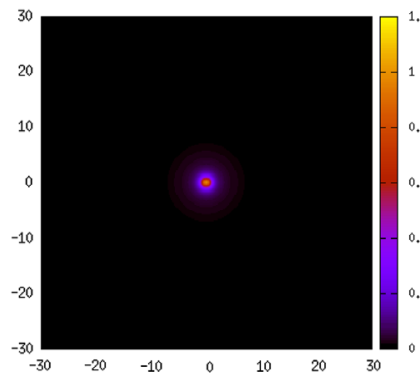

(b)

To estimate the force between two Alice axion strings, we take a large distance approximation, following Ref. [59] for the same problem in the context of non-Abelian strings in dense QCD [60]. Suppose that there exist two heavy Alice strings, which do not move, on the $x$-axis in the $x-y$ plane at a large distance of $2 a$ apart as shown in Fig. 4. So the fields are approximately written as

$$
\begin{aligned}
\Phi & \sim e^{i\left(\theta_{1}+\theta_{2}\right)} P e^{i g \int_{0}^{\theta} \mathbf{A} \cdot \mathbf{d l}}\langle\Phi\rangle P e^{-i g \int_{0}^{\theta} \mathbf{A} \cdot \mathbf{d l} \mathbf{l}}, \\
A_{i}(x, y) & \sim A_{i}^{(+)}(x, y)+A_{i}^{(-)}(x, y)(i=1,2), \\
A_{0} & =A_{3}=0,
\end{aligned}
$$

where $A_{i}^{(+)}\left(A_{i}^{(-)}\right)$is the gauge fields relevant to the positive flux (negative flux) inside the Alice string in the absence of another string with the negative flux (positive flux). So the energy at large distances can be approximately written by

$\mathcal{E} \simeq \int d^{2} x \operatorname{Tr}\left|\mathcal{D}_{i} \Phi\right|^{2} \simeq 2 \eta^{2} \int d^{2} x\left[\partial_{i}\left(\theta_{1}+\theta_{2}\right)\right]^{2}+\cdots$

Here we neglected a small contribution from the gauge field. It is found also that $\int d^{2} x\left[\left(\partial_{i} \theta_{1}\right)^{2}+\left(\partial_{i} \theta_{2}\right)^{2}\right] \simeq$ $2 \times T_{\text {Alice }}$. So the interaction energy can be expressed as

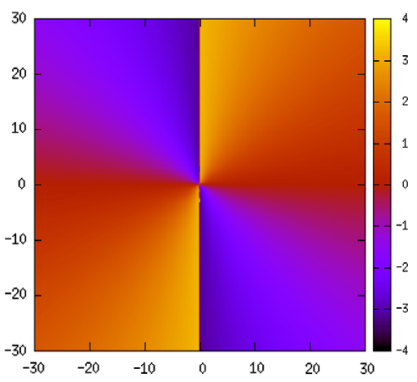

(c)

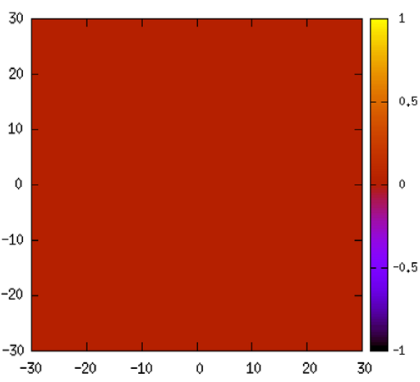

(d)

FIG. 5. Figures of an Abelian axion string before the splitting for $g=1, \eta=0.5, \lambda_{1}=2.0$ and $\lambda_{2}=0.25$. $\operatorname{They} \operatorname{show} 2 \operatorname{Tr}\left(\left(\Phi^{2}\right)^{\dagger} \Phi^{2}\right)$, the static energy, the phase $\Im \log \operatorname{Tr} \Phi^{2}(x, y)$ and non-Abelian magnetic field $F_{12}^{3}(x, y)$ (with a gauge fixing) from left to right. It is easy to check there is a phase jump by $2 \pi$ around each string solution.

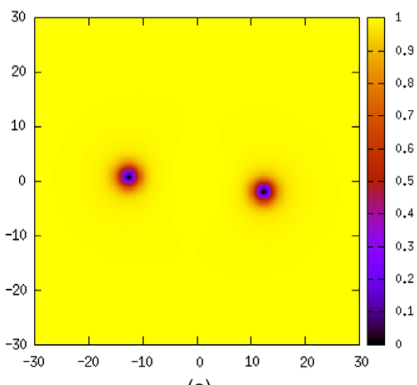

(a)

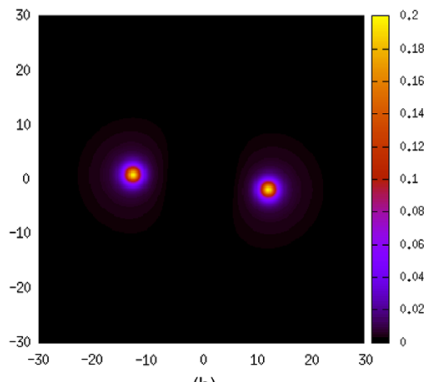

(b)

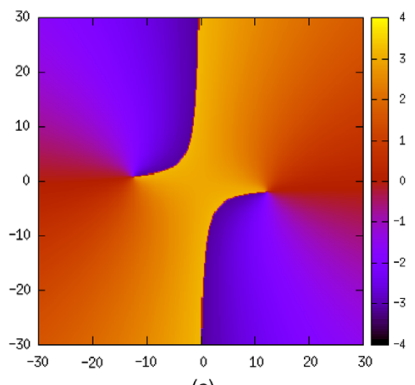

(c)

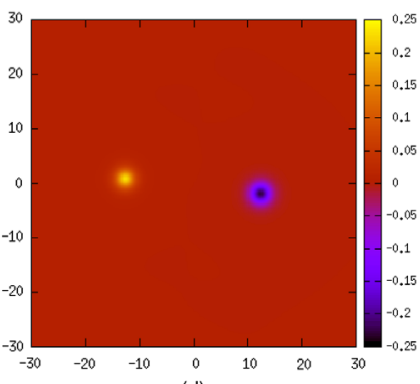

(d)

FIG. 6. Similar figures of two Alice axion strings after the decay from the parent Abelian axion string. 


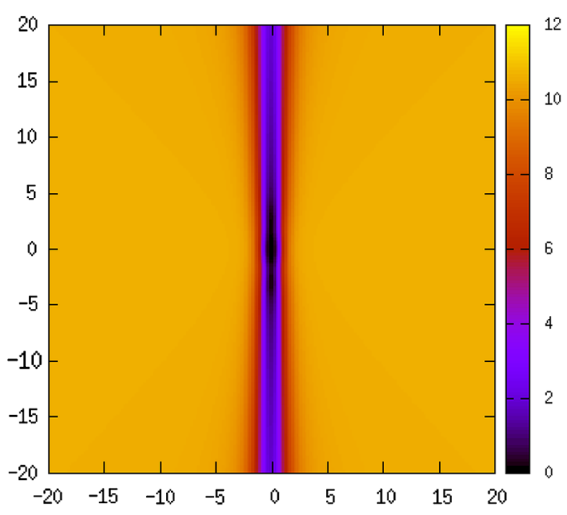

(a)

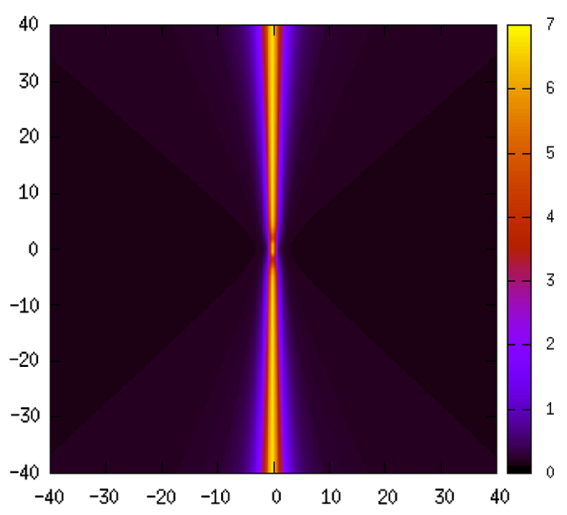

(b)

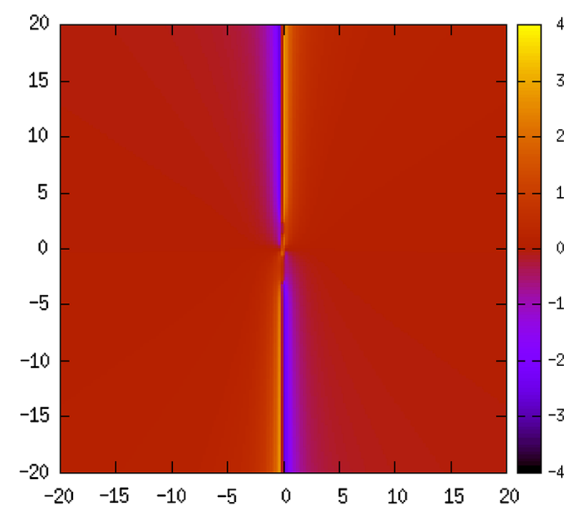

(c)

FIG. 7. Figures of two domain walls attached to one Abelian axion string before the decay for $\mu=1.4, g=1, \eta=0.5, \lambda_{1}=2.0$ and $\lambda_{2}=0.25$. The string exists at the center in each figures. They show: (a) $2 \operatorname{Tr}\left(\left(\Phi^{2}\right)^{\dagger} \Phi^{2}\right)$, (b) the energy density, and (c) the phase $2 \alpha$. It is noted that the $\alpha$ changes by $\pi$ at crossing the domain wall.

$$
\begin{aligned}
\mathcal{E}_{\mathrm{int}} & \simeq 4 \eta^{2} \int d^{2} x \partial_{i} \theta_{1} \partial_{i} \theta_{2}+\cdots \\
& =4 \eta^{2} \cdot 2 \pi \int d r r\left[\frac{r^{2}-a^{2}}{r^{4}+a^{4}-2 r^{2} a^{2} \cos 2 \theta}\right] \\
& \simeq 4 \pi \eta^{2} \log \left(\frac{\rho^{2}+a^{2}}{a^{2}}\right) .
\end{aligned}
$$

Here we used $\partial_{i} \theta=-\epsilon_{i j} x^{j} / r^{2}$ and this is similar to $\theta_{1,2}$. The force between two Alice axion strings is found to be repulsive:

$$
\text { Force }=-\frac{1}{2} \frac{\partial \mathcal{E}_{\text {int }}}{\partial a} \simeq+\frac{4 \pi \eta^{2}}{a} \text { for } \rho \rightarrow \infty
$$

This repulsive force is mediated by the light QCD axion at a large distance apart. ${ }^{7}$ This is analogous to a Coulomb force between particles with the same charge in two spatial dimensions. Hence, the distance between two Alice axion strings would increase with time and it is confirmed from numerical calculation shown in Figs. 5 and 6. However, note that our simulation is done in a relaxation method but not in a real time dynamics.

For numerical solutions, we used $500 \times 500$ lattice with lattice spacing 0.2 . We relaxed the system in 1000000 time steps with each time step $\Delta t=10^{-3}$. The other parameters are taken as $g=1, \eta=0.5, \lambda_{1}=2.0$ and $\lambda_{2}=0.25$.

\footnotetext{
${ }^{7}$ There exists an attractive force mediated by the massive gauge field between two Alice strings at a short distance. It is expected, however, that such Abelian strings tend to decay into Alice strings in the presence of perturbations in the universe. Even if Abelian axion strings survive until the chiral phase transition, they are attached by domain walls and can be split into Alice strings owing to the domain wall tension as shown in Sec. V.
}

\section{DOMAIN WALL-STRING COMPOSITE}

So far we discussed two types of axion strings and decay of a Abelian axion string to two Alice axion strings. In this section, we study configuration of domain walls attached to the Abelian axion string or to the Alice string. The former situation involving the Abelian strings may be realized at the chiral phase transition through the Kibble-Zurek mechanism [46,47]: At the chiral phase transition, two kinds of domain walls may be created elsewhere. If these domain walls collide, they can be glued along an Abelian axion string. The latter situation involving the Alice strings will always take place in our case. To find the domain walls attached to an Abelian axion string, we start with $\mu \neq 0$ in the potential of Eq. (20). The static Hamiltonian density (in $A_{0}=0$ gauge) is given by

$$
\begin{aligned}
\mathcal{H}= & \frac{1}{2} \operatorname{Tr} F_{i j}^{2}++\operatorname{Tr}\left|D_{i} \Phi\right|^{2}+\frac{\lambda_{1}}{2}\left(\operatorname{Tr} \Phi^{\dagger} \Phi-2 \eta^{2}\right)^{2} \\
& +\frac{\lambda_{2}}{2} \operatorname{Tr}\left(\left[\Phi, \Phi^{\dagger}\right]^{2}\right)+\mu\left(\operatorname{Det} \Phi+\operatorname{Det} \Phi^{\dagger}\right)+\text { const. }
\end{aligned}
$$

We shall first discuss vacua in the presence of Abelian axion strings and the Alice axion strings and focus a parameter region, in which $2 \eta^{2} \lambda_{1}>\mu$ is satisfied, for $V_{\mathrm{DW}}$ not to affect $\langle\Phi\rangle$ significantly. This is natural for the axion domain wall since $\mu$ is expected to be of order $m_{a}^{2}$.

\section{A. Abelian axion string-domain wall composite}

First let us consider an Abelian axion string. Below we show that a single Abelian axion string is attached by two domain walls. To understand a situation in the presence of the walls attached to the Abelian axion string, we shall consider an approximate ansatz of the string at a large distance as

$$
\Phi \sim 2 \eta e^{i \alpha} \tau^{1}, \quad A_{\mu}=0 .
$$

Substituting the above ansatz into Eq. (43), we find 


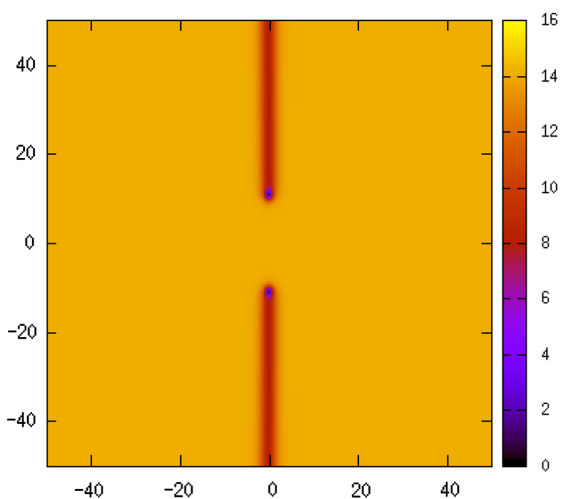

(a)

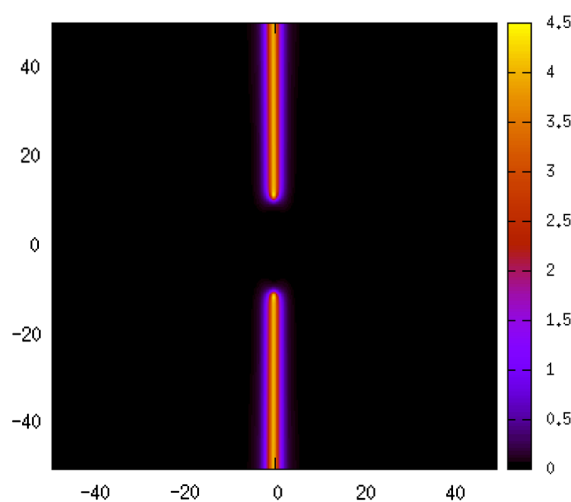

(b)

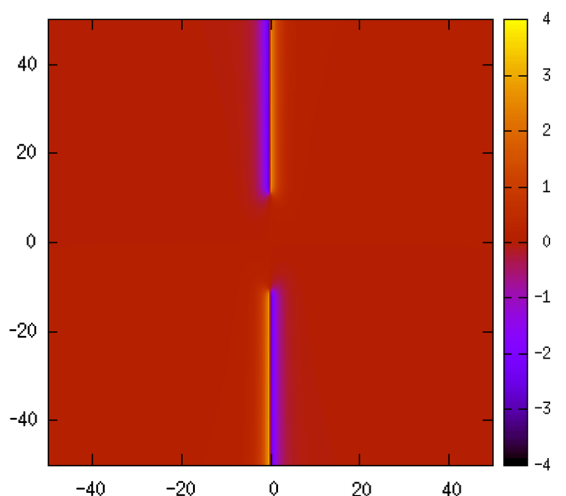

(c)

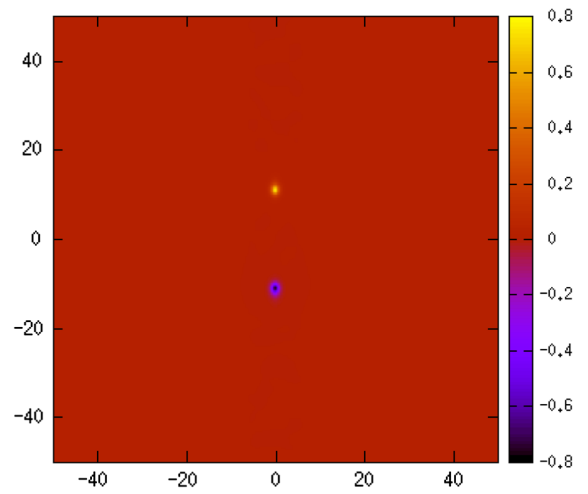

(d)

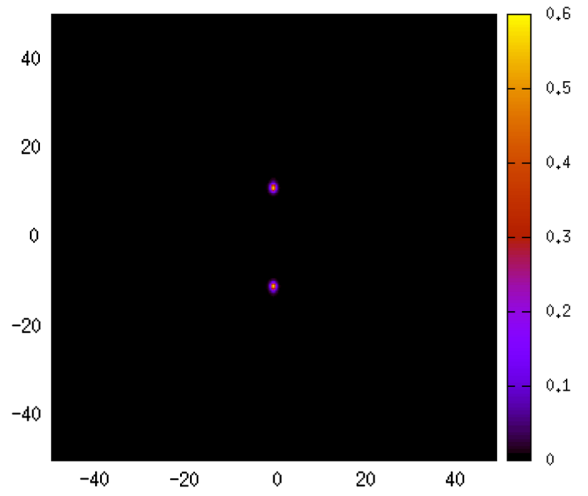

(e)

FIG. 8. Figures of two domain walls attached to two each Alice axion strings after the decay of the parent Abelian axion string for $\mu=1.4, g=1, \eta=0.5, \lambda_{1}=2.0$ and $\lambda_{2}=0.25$. They show: (a) $2 \operatorname{Tr}\left(\left(\Phi^{2}\right)^{\dagger} \Phi^{2}\right)$, (b) energy density, (c) the phase $\alpha$, (d) non-Abelian magnetic field $F_{12}^{3}(x, y)$, (e) the magnitude of the magnetic fields $\sum_{\alpha} F_{12}^{\alpha 2}$. It is noted that $\alpha$ changes by $2 \pi$ at crossing the domain wall.

$$
\mathcal{H} \sim 2 \eta^{2}\left[\left(\partial_{i} \alpha\right)^{2}+\mu(1-\cos 2 \alpha)\right]
$$

The potential in the above Hamiltonian is nothing but the potential of Eq. (19) with $\alpha=a / 2 \eta$. As noted already, $\alpha$ sweeps full circle $(0 \leq \alpha \leq 2 \pi)$ around the axion string, however, the potential shows that the system has two vacua at $\alpha=0$ or $\pi$ and this would create two domain walls attached to the Abelian axion string: $N_{\mathrm{DW}}=2 . \alpha$ would be almost zero or $\pi$ everywhere however changes at the place where domain walls are created. In other words, there are two different domain walls: One is connecting the vacuum at $\alpha=0$ and that at $\alpha=\pi$. Another is doing the vacuum at $\alpha=\pi$ and that at $\alpha=2 \pi \equiv 0$. We call them DW1 and DW2 respectively. The whole configuration can be regarded as a junction of these two domain walls (DW1 and DW2), whose junction line is nothing but an Abelian axion string. Figure 7 shows a full numerical simulation of such a configuration, obtained by the relaxation method. We used larger a lattice of $700 \times 700$ points with lattice spacing 0.2 . We have taken $g=1, \mu=1.4$, $\eta=0.5, \lambda_{1}=2.0$ and $\lambda_{2}=0.25$ for our computation of domain wall-string composites. Here, we put a large friction around a string to prevent this configuration to decay, as described below.

\section{B. Alice axion string-domain wall composite}

In this subsection we discuss the formation of domain walls in the presence of two Alice strings produced via the decay of the parent Abelian axion string. To understand behavior of the walls, we similarly start with field configurations at a large distance as

$$
\begin{gathered}
\Phi \sim \eta\left(\begin{array}{cc}
0 & e^{i \alpha} \\
1 & 0
\end{array}\right), \quad A_{i} \sim-\frac{1}{2 g} \frac{\epsilon_{i j} x^{j}}{r^{2}} \tau^{3} \\
(i, j=1,2), \quad A_{0}=A_{3}=0 .
\end{gathered}
$$

With this ansatz, the static Hamiltonian density in Eq. (43) reads

$$
\mathcal{H} \sim \eta^{2}\left[\frac{1}{2}\left(\partial_{i} \alpha\right)^{2}+2 \mu(1-\cos \alpha)\right]
$$

Here we may check the difference from Eq. (45). In this case, the vacuum is still at $\alpha=0$ (or $2 \pi$ ) whereas the field range is given by $0 \leq \alpha \leq 2 \pi$. As a result, there will exist only one domain wall attached to one Alice axion string. This is a similar to the vacuum with $N_{\mathrm{DW}}=1$. The model is also identical to the sine-Gordon model in two dimensions and a 


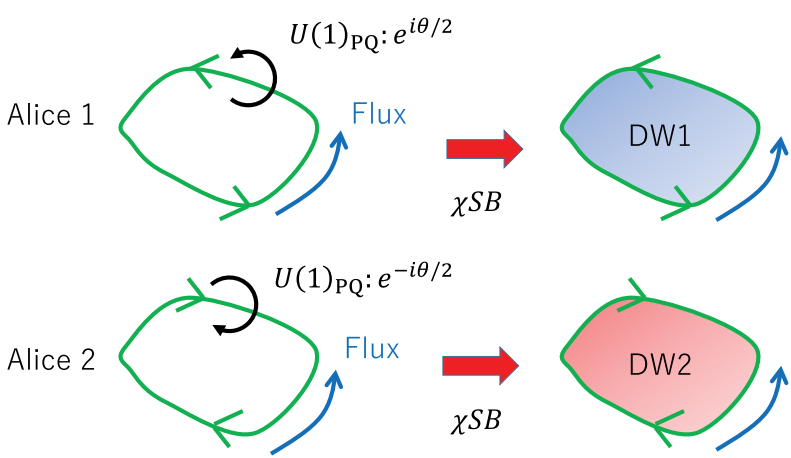

FIG. 9. A schematic figure for Alice strings attached by domain walls. There exist also two kinds of Alice string depending on the orientation and the flux. At the chiral phase transition, DW1 is attached to Alice1 whereas DW2 is attached to Alice2.

domain wall solution along the $x$-axis interpolating between the two vacua can be written as $\alpha=4 \tan ^{-1} e^{ \pm \sqrt{2 \mu} x}$.

For $\mu \neq 0$ in computation, when an Abelian axion string attached by two domain walls is initially created and decays into two Alice axion strings, each domain wall remains attached to Alice strings as shown in Fig. 8. For numerical calculations, the relaxation method is used and the same parameters are chosen as before.

There exist two kinds of the Alice axion strings when one focuses on Eq. (25). One has the non-Abelian flux parallel to the orientation defined by the $U(1)_{\mathrm{PQ}}$ : $e^{i \theta / 2} \Omega=e^{i \theta / 2} e^{i \theta \tau^{3} / 2}$, where $e^{i \theta / 2} \in U(1)_{\mathrm{PQ}}$. Another has the flux opposite to the orientation: $e^{i \theta / 2} \Omega=e^{i \theta / 2} e^{-i \theta \tau^{3} / 2}$ (with a modulus parameter of $\pi$ ). We call these strings Alice1 and Alice2 respectively. To flip the sign simultaneously by $\theta \rightarrow-\theta$ gives the same configuration because this is to see the same string in different ways: the string seen from a positive $z$ coordinate or from a negative $z$ coordinate. So, Alice 2 with $e^{i \theta / 2} e^{-i \theta \tau^{3} / 2}$ is physically equivalent to that with $e^{-i \theta / 2} e^{i \theta \tau^{3} / 2}$. Factors of $e^{ \pm i \theta / 2}$ in $U(1)_{\mathrm{PQ}}$ (with the same $\Omega=e^{i \theta \tau^{3} / 2}$ ) imply the way to approach domain walls in the axion space. After one rotation around the Alice1 with $0 \leq \theta / 2 \leq \pi$, one meets DW1. Further, with $\Omega(2 \pi)=e^{i \pi \tau^{3}} \in \mathbb{Z}_{2} \subset H$, the vacuum is smoothly connected. At the chiral phase transition, like in the $N_{\mathrm{DW}}=1$ case, a single Alice 1 is attached by a single DW1, whereas a single Alice2 is attached by a single DW2. See Fig. 9.

In the actual cosmological history, once an Abelian string is created after the PQ symmetry breaking, it will quickly decay into two Alice strings in the presence of the repulsive force between them. Then domain walls, which are created after the chiral symmetry breaking, attach to Alice strings, and they shrink to a point owing to the wall tension like in the $N_{\mathrm{DW}}=1$ case. Even if an Abelian sting attached by two domain walls is created at the chiral phase transition by the Kibble-Zurek mechanism, the Abelian string can be split into two Alice strings in the presence of the wall tension. Then, one Alice string is attached by one wall and each of the walls similarly shrinks to a point by the tension. See Fig. 10.

\section{CONCLUSION AND DISCUSSIONS}

The axion is an attractive candidate of dark matter, while stable networks composed by stable strings and walls may be created after the breakdown of $U(1)_{\mathrm{PQ}}$ and $\mathbb{Z}_{N_{\mathrm{DW}}}$ embedded in $U(1)_{\mathrm{PQ}}$. They can cause cosmological disasters since the energy density of them can finally dominate that of the universe. The Lazarides-Shafi mechanism is one of the solutions to the domain wall problem. We have studied this mechanism in detail based on a recently proposed model [22], showing dynamics of axion strings and walls. Even if Abelian axion strings are formed in the early universe, each of them is split into multiple Alice axion strings due to a repulsive force between the Alice strings even without domain wall. When domain walls are formed as the universe cools down, a single Alice string is attached by a single wall because a vacuum is connected by a non-Abelian rotation without changing

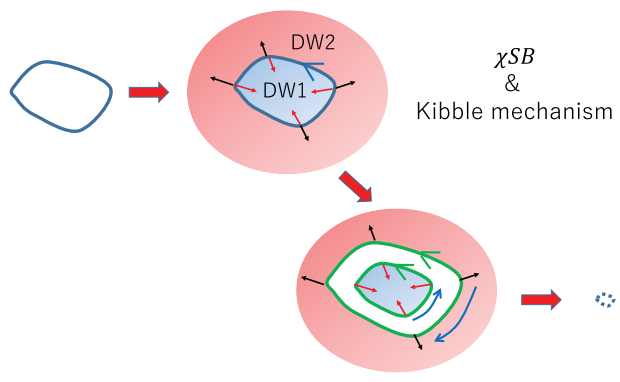

(b)

FIG. 10. Schematic figures for decay of Abelian axion string into two Alice strings attached by domain walls. (a) An Abelian axion string can quickly decay into two Alice strings in the early universe. (b) Even if an Abelian string survives at the chiral phase transition, it can be split into two Alice strings attached by domain walls in the presence of domain wall tension. In both cases, each of the walls shrinks to a point by the tension finally. 
energy. Such walls do not form stable networks since they collapse by the tension of the walls, emitting axions. Even if domain walls attached to the Abelian axion strings are created by the Kibble-Zurek mechanism at the chiral phase transition, the Abelian string can be split into Alice strings and one domain wall is attached to one Alice axion string. Such walls can shrink to a point owing to the wall tension like in the $N_{\text {DW }}=1$ case.

Several discussions are addressed here. The model of Ref. [22] was also proposed as a model for a monopole dark matter. A monopole in the conventional Alice theory [SO (3) gauge theory with scalar fields of the fiveplet] admitting Alice strings was studied in Refs. [61-64]. In particular, a monopole is not spherical and decays into a twisted Alice ring depending on choice of parameters $[62,63]$. It would be interesting to study if the same would happen in our case. In fact, it is known that a global analog (global monopole) shows this property [43]. A dyon with an electric $U(1)_{H}$ charge may be realized as a vorton, namely a persistent electric current flows along a ring. Dyons can be dark matter if their (mini)electric charge, which may be obtained via a kinetic mixing between $U(1)_{H}$ and electromagnetism, is below experimental bounds. It is also worth pointing out that the conventional monopole charge of $\pi_{2}$ is not well defined in the presence of an Alice string, because a monopole becomes an antimonopole when it encircles around an Alice string, as a dual of electric charge encircling around the Alice string. Instead of using the usual homotopy group $\pi_{2}$, a monopole charge must be defined in terms of the Abe homotopy [44].

There can exist infinitely long (Abelian) strings produced after the PQ breaking. In such cases, the scaling solution is found to be violated by a logarithmic growth of the string scaling parameter in time $[65,66]$. It might be hard for wide walls attached to long strings to shrink to a point, hence simulations for them may also be altered. When two Abelian cosmic strings collide, they reconnect each other, which is an important process for cosmic strings to reduce their number. Alice strings have $U(1)$ moduli corresponding to fluxes, and so it is unclear if they can reconnect. In this regard, two non-Abelian strings with
non-Abelian fluxes were shown to always reconnect [67], and so it would be true for Alice strings. Further, a nature of reconnection among Alice strings may be different from that among Abelian strings due to a force existing among Alice strings, so the number of long Alice strings could differ from that of a long Abelian string. The number of long Alice strings would be significant to a solution to the domain wall problem. In any case, the axion abundance needs to be correctly estimated and may be modified from Eq. (9). Thus, an allowed region for the axion decay constant may be altered. If there is no allowed region, the PQ symmetry breaking might be required to take place before or during inflation, and constraint on isocurvature may be important then.

In future observations, gravitational waves produced by the decay of strings and walls may be detected, depending on axion model $[68,69]$. That can be an important signal to verify the presence of axion dark matter produced by the topological objects. In particular, gravitational wave production in the early universe with and without axions can be quite different which should be explored in the future.

If topological objects appear in dark matter models, it is necessary to study the nature of the objects in detail, for precise estimation of dark matter.

\section{ACKNOWLEDGMENTS}

M. N. would like to thank Naoyuki Takeda for his lecture of axion cosmology. This work is supported by the Ministry of Education, Culture, Sports, Science and Technology (MEXT)-Supported Program for the Strategic Research Foundation at Private Universities "Topological Science" (Grant No. S1511006). C. C. acknowledges support as an International Research Fellow of the Japan Society for the Promotion of Science (JSPS) (Grant No. 16F16322). This work is also supported in part by JSPS Grant-in-Aid for Scientific Research [KAKENHI Grants No. 16H03984 (M. N.) and No. 18H01217 (M. N.)], and also by MEXT KAKENHI Grant-in-Aid for Scientific Research on Innovative Areas "Topological Materials Science" No. $15 \mathrm{H} 05855$ (M. N.).
[1] C. A. Baker et al., An Improved Experimental Limit on the Electric Dipole Moment of the Neutron, Phys. Rev. Lett. 97, 131801 (2006).

[2] R. D. Peccei and H. R. Quinn, $C P$ Conservation in the Presence of Instantons, Phys. Rev. Lett. 38, 1440 (1977).

[3] R. D. Peccei and H. R. Quinn, Constraints imposed by $C P$ conservation in the presence of instantons, Phys. Rev. D 16, 1791 (1977).
[4] S. Weinberg, A New Light Boson? Phys. Rev. Lett. 40, 223 (1978).

[5] G. G. Raffelt, Astrophysical axion bounds, Lect. Notes Phys. 741, 51 (2008).

[6] J. Preskill, M. B. Wise, and F. Wilczek, Cosmology of the invisible axion, Phys. Lett. B 120B, 127 (1983).

[7] L. F. Abbott and P. Sikivie, A cosmological bound on the invisible axion, Phys. Lett. 120B, 133 (1983). 
[8] M. Dine and W. Fischler, The not so harmless axion, Phys. Lett. 120B, 137 (1983).

[9] J. E. Kim and G. Carosi, Axions and the strong CP problem, Rev. Mod. Phys. 82, 557 (2010), and references therein; Erratum, Rev. Mod. Phys. 91, 049902 (2019).

[10] M. Kawasaki and K. Nakayama, Axions: Theory and cosmological role, Annu. Rev. Nucl. Part. Sci. 63, 69 (2013), and references therein.

[11] T. W. B. Kibble, G. Lazarides, and Q. Shafi, Walls bounded by strings, Phys. Rev. D 26, 435 (1982).

[12] A. Vilenkin and A. E. Everett, Cosmic Strings and Domain Walls in Models with Goldstone and Pseudo-Goldstone Bosons, Phys. Rev. Lett. 48, 1867 (1982).

[13] A. E. Everett and A. Vilenkin, Left-right symmetric theories and vacuum domain walls and strings, Nucl. Phys. B207, 43 (1982).

[14] Y. B. Zeldovich, I. Y. Kobzarev, and L. B. Okun, Cosmological consequences of the spontaneous breakdown of discrete symmetry, Zh. Eksp. Teor. Fiz. 67, 3 (1974) [Sov. Phys. JETP 40, 1 (1974)].

[15] J. Preskill and A. Vilenkin, Decay of metastable topological defects, Phys. Rev. D 47, 2324 (1993).

[16] Y. Akrami et al. (Planck Collaboration), Planck 2018 results. X. Constraints on inflation, arXiv:1807.06211.

[17] M. Kawasaki, K. Saikawa, and T. Sekiguchi, Axion dark matter from topological defects, Phys. Rev. D 91, 065014 (2015).

[18] V. B. Klaer and G. D. Moore, The dark-matter axion mass, J. Cosmol. Astropart. Phys. 11 (2017) 049.

[19] A. Vilenkin, Gravitational field of vacuum domain walls and strings, Phys. Rev. D 23, 852 (1981); P. Sikivie, Of Axions, Domain Walls and the Early Universe, Phys. Rev. Lett. 48, 1156 (1982); G. B. Gelmini, M. Gleiser, and E. W. Kolb, Cosmology of biased discrete symmetry breaking, Phys. Rev. D 39, 1558 (1989).

[20] G. Lazarides and Q. Shafi, Axion models with no domain wall problem, Phys. Lett. 115B, 21 (1982).

[21] M. Kawasaki, F. Takahashi, and M. Yamada, Suppressing the QCD axion abundance by hidden monopoles, Phys. Lett. B 753, 677 (2016).

[22] R. Sato, F. Takahashi, and M. Yamada, Unified origin of axion and monopole dark matter, and solution to the domain-wall problem, Phys. Rev. D 98, 043535 (2018).

[23] M. Kawasaki, M. Yamada, and T. T. Yanagida, Observable dark radiation from a cosmologically safe QCD axion, Phys. Rev. D 91, 125018 (2015).

[24] A. A. Abrikosov, On the magnetic properties of superconductors of the second group, Zh. Eksp. Teor. Fiz. 32, 1442 (1957) [Sov. Phys. JETP 5, 1174 (1957)].

[25] H. B. Nielsen and P. Olesen, Vortex line models for dual strings, Nucl. Phys. B61, 45 (1973).

[26] M. B. Hindmarsh and T. W. B. Kibble, Cosmic strings, Rep. Prog. Phys. 58, 477 (1995).

[27] A. Vilenkin and E. P. S. Shellard, Cosmic Strings and Other Topological Defects, Cambridge Monographs on Mathematical Physics (Cambridge University Press, Cambridge, England, 2000).

[28] A. S. Schwarz, Field theories with no local conservation of the electric charge, Nucl. Phys. B208, 141 (1982).
[29] J. E. Kiskis, Disconnected gauge groups and the global violation of charge conservation, Phys. Rev. D 17, 3196 (1978).

[30] M. G. Alford, K. Benson, S. R. Coleman, J. March-Russell, and F. Wilczek, The Interactions and Excitations of NonAbelian Vortices, Phys. Rev. Lett. 64, 1632 (1990); Erratum, Phys. Rev. Lett. 65, 668 (1990).

[31] M. G. Alford, K. Benson, S. R. Coleman, J. March-Russell, and F. Wilczek, Zeromodes of non-Abelian vortices, Nucl. Phys. B349, 414 (1991).

[32] M. G. Alford, K. M. Lee, J. March-Russell, and J. Preskill, Quantum field theory of non-Abelian strings and vortices, Nucl. Phys. B384, 251 (1992).

[33] J. Preskill and L. M. Krauss, Local discrete symmetry and quantum mechanical hair, Nucl. Phys. B341, 50 (1990).

[34] M. Bucher and A. Goldhaber, SO(10) cosmic strings and SU(3)-color Cheshire charge, Phys. Rev. D 49, 4167 (1994).

[35] M. Bucher, H. K. Lo, and J. Preskill, Topological approach to Alice electrodynamics, Nucl. Phys. B386, 3 (1992).

[36] H. K. Lo and J. Preskill, Non-Abelian vortices and nonAbelian statistics, Phys. Rev. D 48, 4821 (1993).

[37] J. Striet and F. A. Bais, Simple models with Alice fluxes, Phys. Lett. B 497, 172 (2001).

[38] E. B. Bogomolny, Stability of classical solutions, Yad. Fiz. 24, 861 (1976) [Sov. J. Nucl. Phys. 24, 449 (1976)].

[39] M. K. Prasad and C. M. Sommerfield, An Exact Classical Solution for the 't Hooft Monopole and the Julia-Zee Dyon, Phys. Rev. Lett. 35, 760 (1975).

[40] C. Chatterjee and M. Nitta, BPS Alice strings, J. High Energy Phys. 09 (2017) 046.

[41] C. Chatterjee and M. Nitta, The effective action of a BPS Alice string, Eur. Phys. J. C 77, 809 (2017).

[42] U. Leonhardt and G. E. Volovik, How to create Alice string (half quantum vortex) in a vector Bose-Einstein condensate, Pis'ma Zh. Eksp. Teor. Fiz. 72, 66 (2000) [JETP Lett. 72, 46 (2000)].

[43] J. Ruostekoski and J. R. Anglin, Monopole Core Instability and Alice Rings in Spinor Bose-Einstein Condensates, Phys. Rev. Lett. 91, 190402 (2003); Erratum, Phys. Rev. Lett. 97, 069902 (2006).

[44] S. Kobayashi, M. Kobayashi, Y. Kawaguchi, M. Nitta, and M. Ueda, Abe homotopy classification of topological excitations under the topological influence of vortices, Nucl. Phys. B856, 577 (2012).

[45] Y. Kawaguchi and M. Ueda, Spinor Bose-Einstein condensates, Phys. Rep. 520, 253 (2012).

[46] T. W. B. Kibble, Topology of cosmic domains and strings, J. Phys. A 9, 1387 (1976).

[47] W.H. Zurek, Cosmological experiments in superfluid Helium? Nature (London) 317, 505 (1985).

[48] J. E. Kim, Weak Interaction Singlet and Strong CP Invariance, Phys. Rev. Lett. 43, 103 (1979).

[49] M. A. Shifman, A. I. Vainshtein, and V. I. Zakharov, Can confinement ensure natural $C P$ invariance of strong interactions? Nucl. Phys. B166, 493 (1980).

[50] M. Dine, W. Fischler, and M. Srednicki, A simple solution to the strong $C P$ problem with a harmless axion, Phys. Lett. 104B, 199 (1981).

[51] A. R. Zhitnitsky, On possible suppression of the axion hadron interactions, Yad. Fiz. 31, 497 (1980) [Sov. J. Nucl. Phys. 31, 260 (1980)]. 
[52] T. Hiramatsu, M. Kawasaki, K. Saikawa, and T. Sekiguchi, Axion cosmology with long-lived domain walls, J. Cosmol. Astropart. Phys. 01 (2013) 001.

[53] E. Witten, Dyons of charge $e \theta / 2 \pi$, Phys. Lett. 86B, 283 (1979).

[54] E. Del Nobile, M. Nardecchia, and P. Panci, Millicharge or decay: A critical take on minimal dark matter, J. Cosmol. Astropart. Phys. 04 (2016) 048.

[55] D. S. Akerib et al. (LUX Collaboration), Results from a Search for Dark Matter in the Complete LUX Exposure, Phys. Rev. Lett. 118, 021303 (2017).

[56] W. Fischler and J. Preskill, Dyon-axion dynamics, Phys. Lett. 125B, 165 (1983).

[57] Y. Nomura, S. Rajendran, and F. Sanches, Axion Isocurvature and Magnetic Monopoles, Phys. Rev. Lett. 116, 141803 (2016).

[58] M. Kawasaki, F. Takahashi, and M. Yamada, Adiabatic suppression of the axion abundance and isocurvature due to coupling to hidden monopoles, J. High Energy Phys. 01 (2018) 053.

[59] E. Nakano, M. Nitta, and T. Matsuura, Non-Abelian strings in high density QCD: Zero modes and interactions, Phys. Rev. D 78, 045002 (2008).

[60] M. Eto, Y. Hirono, M. Nitta, and S. Yasui, Vortices and other topological solitons in dense quark matter, Prog. Theor. Exp. Phys. 2014, 012D01 (2014).
[61] R. Shankar, More SO(3) monopoles, Phys. Rev. D 14, 1107 (1976).

[62] F. A. Bais and J. Striet, On a core instability of 't HooftPolyakov monopoles, Phys. Lett. B 540, 319 (2002).

[63] J. Striet and F. A. Bais, More on core instabilities of magnetic monopoles, J. High Energy Phys. 06 (2003) 022.

[64] K. M. Benson and T. Imbo, Topologically Alice strings and monopoles, Phys. Rev. D 70, 025005 (2004).

[65] M. Gorghetto, E. Hardy, and G. Villadoro, Axions from strings: The attractive solution, J. High Energy Phys. 07 (2018) 151.

[66] M. Kawasaki, T. Sekiguchi, M. Yamaguchi, and J. Yokoyama, Long-term dynamics of cosmological axion strings, Prog. Theor. Exp. Phys. 2018, $091 \mathrm{E} 01$ (2018).

[67] M. Eto, K. Hashimoto, G. Marmorini, M. Nitta, K. Ohashi, and W. Vinci, Universal Reconnection of NonAbelian Cosmic Strings, Phys. Rev. Lett. 98, 091602 (2007).

[68] For a recent review see e.g., K. Saikawa, A review of gravitational waves from cosmic domain walls, Universe $\mathbf{3}$, 40 (2017).

[69] T. Higaki, K. S. Jeong, N. Kitajima, T. Sekiguchi, and F. Takahashi, Topological defects and nano-Hz gravitational waves in aligned axion models, J. High Energy Phys. 08 (2016) 044. 